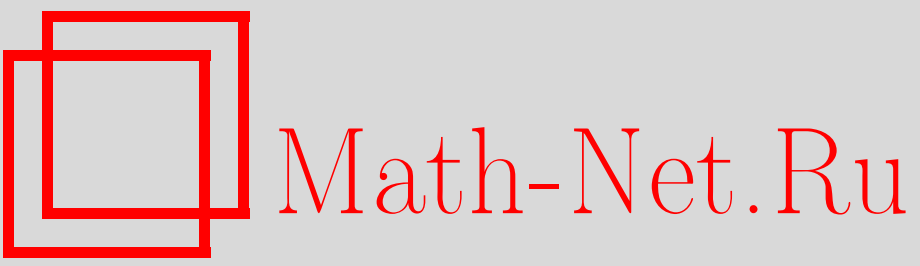

А. Н. Лезнов, Новый подход к теории представлений полупростых алгебр Ли и квантовых алгебр, ТМФ, 2000, том 123, номер 2, 264-284

DOI: https://doi.org/10.4213/tmf601

Использование Общероссийского математического портала Math-Net.Ru подразумевает, что вы прочитали и согласны с пользовательским соглашением

http://www.mathnet.ru/rus/agreement

Параметры загрузки:

IP: 107.22 .136 .117

26 апреля 2023 г., 13:20:48 


\section{НОВЫЙ ПОДХОД К ТЕОРИИ ПРЕДСТАВЛЕНИЙ ПОЛУПРОСТЫХ АЛГЕБР ЛИ И КВАНТОВЫХ АЛГЕБР}

Предложен метод явного построения генераторов простых корней в произвольном конечномерном представлении полупростой квантовой алгебры или алгебры Ли. Метод основан на общих результатах глобальной теории представлений полупростых групп. В качестве примеров рассмотрены алгебры ранга два $A_{2}, B_{2}=C_{2}, D_{2}$ и $G_{2}$. Генераторы простых корней представлены как решения системы конечно-разностных уравнений и даются $\left(N_{l} \times N_{l}\right)$-матрицами, где $N_{l}$ - размерность представления.

\section{1. ВВЕДЕНИЕ}

Базисные векторы неприводимого конечномерного представления полупростой алгебры с весом $l=\left(l_{1}, l_{2}, \ldots, l_{r}\right)$ обычно строятся с помощью многократного применения понижающих генераторов $X_{i}^{-}$к старшему вектору $|l\rangle$, обладающему свойствами

$$
X_{s}^{+}|l\rangle=0, \quad h_{s}|l\rangle=l_{s}|l\rangle
$$

где $X_{s}^{ \pm}$- генераторы, соответствуюшие простым корням, а $h_{s}$ - элементы Картана. Очевидная проблема, связанная с таким построением, заключается в том, что не все векторы состояния, появляющиеся таким образом, линейно независимы, и поэтому требуются дополнительная процедура исключения линейно зависимых компонент и дальнейшая ортогонализация базиса. Обычно сделать это не просто. Тем не менее значения, принимаемые групповым элементом $\exp \tau \equiv \exp \sum h_{i} \tau_{i}$ на базисных векторах, можно получить из инвариантной формулы Вейля для характеров неприводимого представления с весом $l=\sum h_{i} l_{i}$ :

$$
\pi^{l}(\exp \tau)=\frac{\sum_{W} \delta_{W} \exp \left(\tau_{W}, l+\frac{1}{2} \rho\right)}{\sum_{W} \delta_{W} \exp \left(\tau_{W}, \frac{1}{2} \rho\right)} .
$$

Представив это выражение как сумму экспонент

$$
\pi^{l}(\exp \tau)=\sum_{n^{k}}^{N_{l}} C_{n^{k}} \exp \left(\tau, n^{k}\right)=\sum_{n^{k}}^{N_{l}} C_{n^{k}} \exp \sum_{i}^{r}\left(\tau_{i} n_{i}^{k}\right),
$$

\footnotetext{
* Институт физики высоких энергий, Протвино, Московская обл., Россия; Лаборатория теоретической физики им. Н.Н. Боголюбова, ОИЯИ, Дубна, Московская обл., Россия. Е-mail: leznov@ce.ifisicam.unam.mx
} 
можно получить ответы на многие вопросы о структуре базиса соответствующего представления. В формуле (1.1) $W$ - элемент дискретной группы Вейля, $\delta_{W}$ - его сигнатура, $\tau_{W}$ - результат действия $W$ на $\tau, C_{n^{k}}$ - кратность соответствуюшей экспоненты, $\rho-$ сумма положительных корней соответствующей алгебры.

Пусть $K$ - матрица Картана рассматриваемой алгебры Ли и $X_{j}^{-}$- понижающие генераторы. Тогда имеет место соотношение

$$
e^{\tau}\left(X_{1}^{-}\right)^{m_{1}} \ldots\left(X_{r}^{-}\right)^{m_{r}}|l\rangle=e^{\sum \tau_{i} l_{i}} e^{-\sum_{p, s} m_{p} K_{p, s} \tau_{s}}\left(X_{1}^{-}\right)^{m_{1}} \ldots\left(X_{r}^{-}\right)^{m_{r}}|l\rangle
$$

(конечно, порядок понижающих операторов в этом выражении не важен). Приравнивая каждую экспоненту в формуле Вейля к соответствуюшей экспоненте в (1.2), мы легко находим индексы $m_{i}$. Единственное, чего нельзя сделать с помошью формулы Вейля, это различить базисные векторы, появляющиеся из одинаковых наборов понижающих операторов, взятых в различном порядке. Однако число таких состояний определяется кратностью соответствуюших экспонент в формуле для характера.

В этой работе приведенные выше замечания играют ключевую роль. Мы предлагаем альтернативный подход к явному построению генераторов простых корней. Вместо процедуры исключения линейно зависимых компонент и последующей ортогонализации нам требуется решить систему конечно-разностных уравнений, разрешимость которой гарантируется глобальной теорией представлений полупростых алгебр. В принципе эти условия можно найти независимо (как условия разрешимости такой системы с “фиксированной” границей), но можно и убедиться в их эквивалентности известным результатам глобальной теории представлений.

Вкратце мы предлагаем следуюшую программу. Начальные данные - это известные размерности и характеры неприводимых представлений полупростых групп, задаваемые знаменитой формулой Вейля [1]. Конечные данные - это явный вид генераторов простых корней как квантовых полупростых алгебр, так и полупростых алгебр Ли. Мы не делаем различия между базисами для квантовых полупростых алгебр и полупростых алгебр Ли и представляем в явной форме генераторы простых корней в виде $\left(N_{l} \times N_{l}\right)$-матриц $\left(N_{l}\right.$ - размерность соответствующего представления, задаваемая формулой размерностей Вейля).

В разделе 2 мы переписываем определяющие квантовую алгебру соотношения в терминах только $2 r$ генераторов вместо $3 r$ генераторов, используемых в традиционном подходе. Эта конструкция конкретизируется в разделе 3 для алгебр ранга 2. В разделах 4 , 5,6 представлены детальные вычисления для алгебр $A_{2}, B_{2}=C_{2}$ и $G_{2}$. В разделе 7 содержатся заключительные замечания и обсуждаются перспективы дальнейших исследований.

Ниже мы не собираемся строить теорию квантовых алгебр каким-то новым способом. Мы только используем многие результаты стандартной теории с целью получить явный вид генераторов простых корней в данном конечномерном представлении. Мы выбираем только те факты, которые важны для нашей цели. По этой причине не упоминаются соотношения Серра (в связи с уравнениями (2.1) (см. ниже) важные для определения явного вида матриц Картана), а непосредственно рассматриваем (2.1) как коммутационные соотношения для данной полупростой серии (с данной матрицей Картана), которые 
мы хотим разрешить. Будем считать известным, что базис конечномерного неприводимого представления в квантовом случае можно выбрать таким же, как и в полупростой алгебре Ли. В этом базисе генераторы можно выбрать так, что они будут удовлетворять дополнительным условиям $X_{i}^{+}=\left(X_{i}^{-}\right)^{T}, h_{i}=\left(h_{i}\right)^{T}$ с диагональными $h_{i}$. В этом базисе операторы $Q=\exp \left(h_{1}+p h_{2}\right)$ также диагональны, а из соотношений (3.6) (см. ниже) следуют обычные правила отбора для ненулевых матричных элементов матриц $s^{1}$ и $s^{2}$.

\section{2. МОДИФИЦИРОВАННАЯ ФОРМА УРАВНЕНИЙ ДЛЯ КВАНТОВЫХ АЛГЕБР}

В обычной форме $3 r^{2}$ коммутационных соотношений между $3 r$ генераторами квантовой алгебры (генераторами $X_{i}^{ \pm}$, соответствуюшими простым корням, и элементами Картана $\left.h_{i}\right)$ имеют вид

$$
\left[h_{i}, X_{j}^{ \pm}\right]= \pm K_{j, i} X_{j}^{ \pm}, \quad\left[X_{i}^{+}, X_{j}^{-}\right]=\delta_{j, i} \frac{\operatorname{sh}\left(t w_{i} h_{i}\right)}{\operatorname{sh}\left(t w_{i}\right)}
$$

где $K$-матрица Картана, $K_{j, i} w_{i}=w_{j} K_{j, i} \equiv \widetilde{K}_{j, i}, t$-параметр деформации. Матрицы Картана серий $A_{n}, D_{n}$ и $E_{6,7,8}$ являются априори симметричными, при этом $\widetilde{K}_{i, j}=$ $K_{i, j}$, a $w_{i}=1$.

Введем множество $3 r$ генераторов, альтернативное (2.1):

$$
T_{i}^{ \pm}=e^{\frac{t w_{i} h_{i}}{4}} X_{i}^{ \pm} e^{\frac{t w_{i} h_{i}}{4}}, \quad R_{i}=e^{t w_{i} h_{i}}
$$

В терминах этих генераторов искомые соотношения, определяющие квантовую алгебру (2.1), можно переписать в виде

$$
R_{i} T_{j}^{ \pm}=e^{ \pm \widetilde{K}_{j, i} t} T_{j}^{ \pm} R_{i}, \quad e^{\frac{\widetilde{K}_{j, i} t}{2}} T_{i}^{+} T_{j}^{-}-e^{\frac{-\widetilde{K}_{j, i} t}{2}} T_{j}^{-} T_{i}^{+}=\delta_{i, j} \frac{R_{i}^{2}-1}{2 \operatorname{sh}\left(t w_{i}\right)}
$$

Вводя $2 r$ генераторов

$$
Q_{i}^{ \pm}=T_{i}^{ \pm} \pm \frac{R_{i}}{2 \operatorname{sh}\left(t w_{i}\right)},
$$

перепишем систему (2.2) в виде

$$
\begin{gathered}
e^{t w_{i}} Q_{i}^{+} Q_{i}^{-}-e^{-t w_{i}} Q_{i}^{-} Q_{i}^{+}=-\frac{1}{2 \operatorname{sh}\left(t w_{i}\right)}, \\
e^{\frac{\widetilde{K}_{j, i} t}{2}}\left(Q_{i}^{+} Q_{j}^{-}-Q_{j}^{+} Q_{i}^{-}\right)-e^{\frac{-\widetilde{K}_{j, i} t}{2}}\left(Q_{j}^{-} Q_{i}^{+}-Q_{i}^{-} Q_{j}^{+}\right)=0, \quad \widetilde{K}_{i, j} \neq 0, \\
{\left[Q_{i}^{+}, Q_{j}^{-}\right]=\left[Q_{j}^{+}, Q_{i}^{-}\right]=0, \quad \widetilde{K}_{i, j}=0,} \\
e^{\frac{\widetilde{K}_{j, i} t}{2}}\left(Q_{i}^{+} Q_{j}^{-}+Q_{j}^{+} Q_{i}^{-}\right)-e^{\frac{-\widetilde{K}_{j, i} t}{2}}\left(Q_{j}^{-} Q_{i}^{+}+Q_{i}^{-} Q_{j}^{+}\right)= \\
=-\frac{\operatorname{sh}\left(\frac{\widetilde{K}_{j, i}}{2} t\right)}{\operatorname{sh}\left(t w_{i}\right) \operatorname{sh}\left(t w_{j}\right)} R_{i} R_{j}, \quad \widetilde{K}_{i, j} \neq 0, \quad i \neq j \\
R_{i} Q_{j}^{ \pm}=e^{ \pm \widetilde{K}_{j, i} t} Q_{j}^{ \pm} R_{i} \mp\left(e^{ \pm \widetilde{K}_{j, i} t}-1\right) \frac{R_{j} R_{i}}{2 \operatorname{sh}\left(t w_{j}\right)} .
\end{gathered}
$$


Заметим, что первые три строки связывают генераторы $Q_{i}^{ \pm}$между собой. Следовательно, можно рассматривать эти соотношения как относяшиеся к некоторой подалгебре универсальной обертывающей алгебры $(2.1)^{1)}$.

Покажем, что, как прямо следует из уравнений (2.3), все генераторы $R_{i}$ можно алгебраически выразить через генераторы $Q_{s}^{ \pm}$(при таких действиях возникает только оператор $Q_{i j}^{-1}$, обратный к оператору $Q_{i j}=\exp \left(w_{i} h_{i}+w_{j} h_{j}\right)$, который обратим в каждом конечномерном неприводимом представлении). Для этой цели умножим уравнение из последней строки формулы $(2.3)$ (заменив индексы $i \rightarrow j$ ) на $R_{i}$ слева. Получим

$$
\begin{aligned}
R_{i} R_{j} Q_{i}^{ \pm} & =e^{ \pm \widetilde{K}_{i, j} t} R_{i} Q_{i}^{ \pm} R_{j} \mp\left(e^{ \pm \widetilde{K}_{i j} t}-1\right) \frac{R_{i}^{2} R_{j}}{2 \operatorname{sh}\left(t w_{i}\right)}= \\
& =e^{ \pm\left(\widetilde{K}_{i i}+\widetilde{K}_{i, j} t\right)} Q_{i}^{ \pm} R_{i} R_{j} \mp\left(e^{ \pm\left(\widetilde{K}_{i i}+\widetilde{K}_{i j} t\right)}-1\right) \frac{R_{i} R_{j} R_{i}}{2 \operatorname{sh}\left(t w_{i}\right)} .
\end{aligned}
$$

Вводя оператор

$$
Q_{i, j} \equiv e^{\frac{\widetilde{K}_{i j} t}{2}}\left(Q_{i}^{+} Q_{j}^{-}+Q_{j}^{+} Q_{i}^{-}\right)-e^{-\frac{\widetilde{K}_{i j} t}{2}}\left(Q_{j}^{-} Q_{i}^{+}+Q_{i}^{-} Q_{j}^{+}\right)=Q_{j, i},
$$

который в силу (2.3) пропорционален $R_{i} R_{j}$, мы можем переписать соотношение (2.4) в виде

$$
Q_{i j} Q_{i}^{ \pm} Q_{i j}^{-1}=e^{ \pm\left(\widetilde{K}_{i i}+\widetilde{K}_{i j}\right) t} Q_{i}^{ \pm} \mp\left(e^{ \pm\left(\widetilde{K}_{i i}+\widetilde{K}_{i j}\right) t}-1\right) \frac{R_{i}}{2 \operatorname{sh}\left(t w_{i}\right)} .
$$

Из уравнения (2.5) можно заключить, что при $\widetilde{K}_{i i}+\widetilde{K}_{i j} \neq 0$ приведенное выше утверждение верно и генератор $R_{i}$ можно алгебраически выразить через генераторы $Q^{ \pm}$. Если $\widetilde{K}_{i i}+\widetilde{K}_{i j}=0$, то, несомненно, $\widetilde{K}_{j j}+\widetilde{K}_{i j} \neq 0$ игенератор $R_{j}$ можно выразить в терминах $Q$-генераторов. Тогда $R_{i}$ можно найти из уравнения, связывающего $Q_{i, j}$ с произведением $R_{i} R_{j}$. Таким образом, приведенное выше утверждение верно во всех случаях.

В следующем разделе внимание будет сосредоточено на случае ранга два. Случай ранга один хорошо известен, и мы только приведем основные относяшиеся к нему факты для последуюшего использования. Неприводимые представления алгебры $A_{1}^{q}$ нумеруются натуральным или полунатуральным числом $l$, при этом размерность представления равна $2 l+1$. Собственные значения генератора $H$ равны всем нечетным или четным числам между $2 l$ и $-2 l ; H_{k}=2 l-2 k \equiv 2 m, 0 \leqslant k \leqslant 2 l$. Ненулевые матричные элементы генераторов $X^{ \pm}$имеют вид

$$
X_{m, m \pm 1}^{ \pm}=\left(\frac{\operatorname{sh}[(l \mp m) t]}{\operatorname{sh} t} \frac{\operatorname{sh}[(l \pm m+1) t]}{\operatorname{sh} t}\right)^{\frac{1}{2}},
$$

где $m=l-k$ и выполнено условие $X^{+}=\left(X^{-}\right)^{T}$. Генераторы $Q^{ \pm}$имеют диагональные элементы

$$
Q_{m, m}^{ \pm}=\frac{e^{ \pm 2(l-k) t}}{2 \operatorname{sh} t}
$$

\footnotetext{
1) Для алгебры $A_{1}^{q}$ два генератора $Q^{ \pm}$удовлетворяют одному соотношению $e^{t} Q^{+} Q^{-}-e^{-t} \times$ $Q^{-} Q^{+}=-(2 \operatorname{sh} t)^{-1}$, которое можно рассматривать как некоторую квантовую алгебру со своей собственной теорией представлений.
} 
и ненулевые недиагональные элементы

$$
Q_{m, m \pm 1}^{ \pm}=e^{\left(m \pm \frac{1}{2}\right) t}\left(\frac{\operatorname{sh}[(l \mp m) t]}{\operatorname{sh} t} \frac{\operatorname{sh}[(l \pm m+1) t]}{\operatorname{sh} t}\right)^{\frac{1}{2}}
$$

\section{3. АЛГЕБРЫ РАНГА ДВА}

В этом разделе мы ограничимся только двумя значениями индексов $i=1,2$ в общей системе (2.3) или алгебрами ранга два $A_{2}, B_{2}=C_{2}$ и $G_{2}$. Симметричная матрица Kартана для этих алгебр имеет вид

$$
\widetilde{K}=\left(\begin{array}{cc}
2 & -p \\
-p & 2 p
\end{array}\right), \quad w_{1}=1, \quad w_{2}=p
$$

где $p=1,2,3$ для $A_{2}, B_{2}, G_{2}$ соответственно. Введем обозначения

$$
Q_{1}^{ \pm}=\frac{s^{1} \pm r^{1}}{2 \operatorname{sh} t}, \quad Q_{2}^{ \pm}=\frac{s^{2} \pm r^{2}}{2 \operatorname{sh}(p t)}
$$

Первые две строки в системе (2.3) принимают вид

$$
\begin{aligned}
{\left[s^{1}, r^{1}\right] } & =\operatorname{th} t\left(\left(s^{1}\right)^{2}-\left(r^{1}\right)^{2}+1\right), \\
{\left[s^{2}, r^{2}\right] } & =\operatorname{th}(p t)\left(\left(s^{2}\right)^{2}-\left(r^{2}\right)^{2}+1\right), \\
{\left[s^{1}, s^{2}\right]-\left[r^{1}, r^{2}\right] } & =\operatorname{th}\left(\frac{p t}{2}\right)\left(\left\{r^{1}, s^{2}\right\}-\left\{s^{1}, r^{2}\right\}\right),
\end{aligned}
$$

где $\{$,$\} - антикоммутатор.$

Оператор $Q_{1,2} \equiv Q$ задается формулой (мы используем перенормированный вариант обшего определения из предыдушего раздела)

$$
Q=\frac{\operatorname{sh} t \operatorname{sh}(p t)}{\operatorname{sh}\left(\frac{p t}{2}\right)}\left(e^{\frac{-p t}{2}}\left(Q_{1}^{+} Q_{2}^{-}+Q_{2}^{+} Q_{1}^{-}\right)-e^{\frac{p t}{2}}\left(Q_{1}^{-} Q_{2}^{+}+Q_{2}^{-} Q_{1}^{+}\right)\right)=R_{1} R_{2} .
$$

Операторы $R_{1}$ и $R_{2}$ выражаются через операторы $Q, Q_{1}^{ \pm}, Q_{2}^{ \pm}$с помошью соотоношений

$$
\begin{aligned}
& Q Q_{1}^{ \pm} Q^{-1}=e^{\mp(p-2) t} Q_{1}^{ \pm} \mp\left(e^{\mp(p-2) t}-1\right) \frac{R_{1}}{2 \operatorname{sh} t}, \\
& Q Q_{2}^{ \pm} Q^{-1}=e^{ \pm p t} Q_{2}^{ \pm} \mp\left(e^{ \pm p t}-1\right) \frac{R_{2}}{\operatorname{sh}(p t)} .
\end{aligned}
$$

В терминах $s^{1}, s^{2}$ и $r^{1}, r^{2}$ эти соотношения можно переписать в более подходяшем для наших целей виде

$$
\begin{aligned}
\operatorname{sh}[(p-2) t] R_{1} & =\operatorname{sh}[(p-2) t] r^{1}+Q s^{1} Q^{-1}-\operatorname{ch}[(p-2) t] s^{1}, \\
-\operatorname{sh}(p t) R_{2} & =-\operatorname{sh}(p t) r^{2}+Q s^{2} Q^{-1}-\operatorname{ch}(p t) s^{2} .
\end{aligned}
$$


Заметим, что для алгебры $B_{2}=C_{2}(p=2)$ оператор $R_{1}$ нельзя определить из соотношений (3.3). Однако корректно определен оператор $R_{2}$, после чего оператор $R_{1}$ можно алгебраически выразить из уравнения для $Q$. Исключая $R_{1}$ и $R_{2}$ из четырех уравнений (3.3), получаем

$$
\begin{gathered}
Q\left(e^{\frac{(p-2) t}{2}} Q_{1}^{+}-e^{-\frac{(p-2) t}{2}} Q_{1}^{-}\right) Q^{-1}=\frac{(p-2) t}{2} Q_{1}^{+}-e^{\frac{(p-2) t}{2}} Q_{1}^{-} \\
Q\left(e^{-\frac{p t}{2}} Q_{2}^{+}-e^{\frac{p t}{2}} Q_{2}^{-}\right) Q^{-1}=e^{\frac{p t}{2}} Q_{2}^{+}-e^{-\frac{p t}{2}} Q_{2}^{-}
\end{gathered}
$$

Эти уравнения эквивалентны уравнениям

$$
\begin{gathered}
\operatorname{ch}\left[\frac{(p-2) t}{2}\right]\left(r^{1}-Q r^{1} Q^{-1}\right)=\operatorname{sh}\left[\frac{(p-2) t}{2}\right]\left(s^{1}+Q s^{1} Q^{-1}\right), \\
\operatorname{ch}\left(\frac{p t}{2}\right)\left(r^{2}-Q r^{2} Q^{-1}\right)=-\operatorname{sh}\left(\frac{p t}{2}\right)\left(s^{2}+Q s^{2} Q^{-1}\right) .
\end{gathered}
$$

Прямым следствием уравнений (2.3) является взаимная коммутативность операторов $R_{1}, R_{2}$ и $Q$. Отсюда следуют соотношения

$$
Q s^{1} Q^{-1}+Q^{-1} s^{1} Q=2 \operatorname{ch}[(p-2) t] s^{1}, \quad Q s^{2} Q^{-1}+Q^{-1} s^{2} Q=2 \operatorname{ch}(p t) s^{2},
$$

из которых вытекает, что в представлениях с диагональным $Q$-оператором матричные элементы генераторов матриц $s^{1}$ и $s^{2}$ удовлетворяют уравнениям

$$
\left(\frac{\lambda_{i}}{\lambda_{j}}+\frac{\lambda_{j}}{\lambda_{i}}-e^{(p-2) t}-e^{-(p-2) t}\right) s_{i, j}^{1}=0, \quad\left(\frac{\lambda_{i}}{\lambda_{j}}+\frac{\lambda_{j}}{\lambda_{i}}-e^{p t}-e^{-p t}\right) s_{i, j}^{2}=0,
$$

где $\lambda_{i}$ - собственные значения матришы $Q$. Другими словами, матричные элементы $s_{i, j}^{1}$ отличны от нуля только в случае, если $\lambda_{i} / \lambda_{j}=e^{ \pm(p-2) t}$, и аналогично матричные элементы $s_{i, j}^{2}$ отличны от нуля, если $\lambda_{i} / \lambda_{j}=e^{ \pm p t}$. В последующих разделах мы конкретизируем эти соотношения для трех отдельных случаев алгебр $A_{2}, B_{2}=C_{2}$ и $G_{2}$.

\section{4. АЛГЕБРА $A_{2}$}

Рассматривается базис с диагональным $Q$-генератором. Из уравнения (3.7) (при $p=1$ ) получаем, что $\lambda_{i} / \lambda_{i+1}=e^{t}$ (учитывая неприводимость матриц $s^{1}$ и $r^{1}$ ). Однако ничего нельзя сказать по поводу кратности каждого $\lambda_{i}$. Будем полагать ее произвольной и равной $N_{i}$. Из уравнения (3.6) следует, что генераторы матриц $s^{1}$ и $s^{2}$ имеют блочную структуру

$$
s^{1}=\left(\begin{array}{lllll}
\ldots & a_{i, i-1} & 0 & a_{i, i+1} & \ldots
\end{array}\right), \quad s^{2}=\left(\begin{array}{lllll}
\ldots & b_{i, i-1} & 0 & b_{i, i+1} & \ldots
\end{array}\right),
$$

где $a_{i, i-1}$ и $b_{i, i-1}$ - прямоугольные матрицы размером $N_{i-1} \times N_{i}$, а $a_{i, i+1}, b_{i, i+1}$ - прямоугольные матрицы размером $N_{i} \times N_{i+1}$ (нуль в центре - это нулевая $\left(N_{i} \times N_{i}\right)$-матрица). Из уравнений (3.5) (при $p=1$ ) можно получить блочную структуру матриц $r^{1}$ и $r^{2}$ :

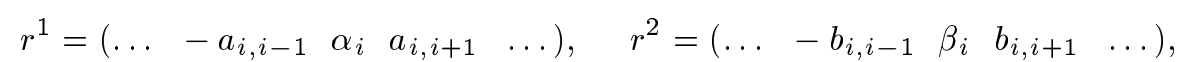


где $\alpha_{i}$ и $\beta_{i}-\left(N_{i} \times N_{i}\right)$-матрицы. Система уравнений, которым удовлетворяют матрицы $a, b, \alpha, \beta$, возникает после подстановки анзацев для $s$ и $r$ в уравнения (3.1) и (3.2). Уравнение, связываюшее $s^{1}$ и $r^{1}$, эквивалентно матричной системе

$$
\begin{gathered}
e^{-t} a_{n, n-1} \alpha_{n-1}=e^{t} \alpha_{n} a_{n, n-1}, \quad e^{t} a_{n, n+1} \alpha_{n+1}=e^{-t} \alpha_{n} a_{n, n+1}, \\
2 e^{-t} a_{n, n-1} a_{n-1, n}-2 e^{t} a_{n, n+1} a_{n+1, n}=\operatorname{sh} t\left(I_{n}-\alpha_{n}^{2}\right) .
\end{gathered}
$$

Аналогичные рассуждения для $s^{2}$ и $r^{2}$ приводят к соотношениям

$$
\begin{gathered}
e^{-t} b_{n, n-1} \beta_{n-1}=e^{t} \beta_{n} b_{n, n-1}, \quad e^{t} b_{n, n+1} \beta_{n+1}=e^{-t} \beta_{n} b_{n, n+1} \\
2 e^{-t} b_{n, n-1} b_{n-1, n}-2 e^{t} b_{n, n+1} b_{n+1, n}=\operatorname{sh} t\left(I_{n}-\beta_{n}^{2}\right)
\end{gathered}
$$

где $I_{n}$ обозначает единичную $\left(N_{n} \times N_{n}\right)$-матрицу. Из последнего из уравнений $(3.1)$ и определения $Q(3.2)$ следует система

$$
\begin{gathered}
e^{\frac{t}{2}} b_{n, n-1} \alpha_{n-1}=e^{-\frac{t}{2}} \alpha_{n} b_{n, n-1}, \quad e^{-\frac{t}{2}} a_{n, n+1} \beta_{n+1}=e^{\frac{t}{2}} \beta_{n} a_{n, n+1}, \\
e^{\frac{t}{2}} a_{n, n-1} \beta_{n-1}=e^{-\frac{t}{2}} \beta_{n} a_{n, n-1}, \quad e^{-\frac{t}{2}} b_{n, n+1} \beta_{n+1}=e^{\frac{t}{2}} \alpha_{n} b_{n, n+1}, \\
2 e^{-\frac{t}{2}}\left(\frac{\alpha_{n} \beta_{n}}{4}+b_{n, n+1} a_{n+1, n}\right)-2 e^{\frac{t}{2}}\left(\frac{\beta_{n} \alpha_{n}}{4}+a_{n, n-1} b_{n-1, n}\right)=\operatorname{sh} \frac{t}{2} \lambda_{n} I_{n} .
\end{gathered}
$$

С первого взглядя система (4.1)-(4.3) производит впечатление настолько сложной, что кажется, что все попытки ее решить имеют мало шансов на успех. Однако, как мы покажем ниже, это не так. Прежде всего подставим анзац для $s^{1}, r^{1}, s^{2}$ и $r^{2}$ в уравнение (3.4) (при $p=1$ ). Прямые вычисления показывают, что $R_{1}$ и $R_{2}$ представляют собой блочно-диагональные матрищы с блочными матрищами $\alpha_{n}$ и $\beta_{n}$ соответственно. Более того, из взаимной коммутативности $R_{1}$ и $R_{2}$ следует, что $\left[\alpha_{n}, \beta_{n}\right]=0$. Уравнение $(3.2)$, связывающее $Q$ с $R_{1}$ и $R_{2}$, можно переписать в виде $\lambda_{n} I_{n}=\alpha_{n} \beta_{n}$. Принимая все это во внимание, исключим матрицы $\beta_{n}$ из системы $(4.1)-(4.3)$ и перепишем эту систему в виде

$$
\begin{aligned}
& e^{-t} a_{n, n-1} \alpha_{n-1}=e^{t} \alpha_{n} a_{n, n-1}, \quad e^{t} a_{n, n+1} \alpha_{n+1}=e^{-t} \alpha_{n} a_{n, n+1} \\
& e^{\frac{t}{2}} b_{n, n-1} \alpha_{n-1}=e^{-\frac{t}{2}} \alpha_{n} b_{n, n-1}, \quad e^{-\frac{t}{2}} b_{n, n+1} \alpha_{n+1}=e^{\frac{t}{2}} \alpha_{n} b_{n, n+1} \\
& 2 e^{-t} a_{n, n-1} a_{n-1, n}-2 e^{t} a_{n, n+1} a_{n+1, n}=\operatorname{sh} t\left(I_{n}-\alpha_{n}^{2}\right) \\
& 2 e^{-t} b_{n, n-1} b_{n-1, n}-2 e^{t} b_{n, n+1} b_{n+1, n}=\operatorname{sh} t\left(I_{n}-\lambda_{n}^{2} \alpha_{n}^{-2}\right) \\
& e^{-\frac{t}{2}} b_{n, n+1} a_{n+1, n}-e^{\frac{t}{2}} a_{n, n-1} b_{n-1, n}=0
\end{aligned}
$$

Как было отмечено выше, матрица $\alpha_{n}$ коммутирует с $\beta_{n}$, так что обе они могут быть одновременно приведены к диагональному виду. Диагональные элементы матриш $\alpha_{n}$ и $\beta_{n}$ обозначим двойными индексами $\alpha_{n}^{s}, \beta_{n}^{s}, 1 \leqslant s \leqslant N_{n}$. Очевидно, что $\alpha_{n}^{s} \beta_{n}^{s}=\lambda_{n}$.

Система (4.4) в представленном виде неограничена, и для ее решения необходимы некоторые дополнительные "граничные" условия. Чтобы получить решение в виде конечномерных матриц, примем, что на “левом конце" $a_{1,0}=b_{1,0}=0$, а на "правом конце" 
$a_{N, N+1}=b_{N, N+1}=0$. Числа $N_{n}$ и диагональные элементы $\lambda_{n}, \alpha_{n}^{s}$ должны быть найдены из условия разрешимости системы (4.4) при этих граничных условиях. Мы будем использовать известные результаты глобальной теории представлений алгебры $A_{2}$, для того чтобы явно решить систему (4.4) и, таким образом, получить явный вид генераторов простых корней для неприводимых представлений $(p, q)$ алгебры $A_{2}$.

Как было упомянуто во введении, базисные векторы неприводимого представления полупростых алгебр строятся из одного старшего вектора путем действия понижающих операторов. Из определения старшего вектора следует, что $\lambda_{1}=e^{(p+q) t}, \alpha_{1}^{1}=e^{p t}$ и $N_{1}=1$. Напомним, что $\alpha=\exp \left(h_{1} t\right)$ и $\lambda=\exp \left[\left(h_{1}+h_{2}\right) t\right]$. Рассмотрим базисные векторы, возникающие после действия генераторов матриц $X_{1}^{-}$и $X_{2}^{-}$на старший вектор. Очевидно, что

$$
N_{2}=2, \quad q \neq 0, \quad \lambda_{2}=e^{(p+q-1) t}, \quad \alpha_{2}^{1}=e^{(p-2) t}, \quad \alpha_{2}^{2}=e^{(p+1) t} .
$$

Временно оставим в стороне задачу о линейно независимых компонентах. Тогда на каждом шаге после применения двух генераторов матриц $X_{1}^{-}$и $X_{2}^{-}$к каждому базисному вектору, полученному на предыдушем шаге, число базисных векторов удваивается по сравнению с предыдушим шагом, и становятся очевидными соотношения

$$
N_{s}=2^{s-1}, \quad \lambda_{s}=e^{(p+q-s+1) t}, \quad \alpha_{s}^{k}=e^{(p-2(s-1)+3 k) t}, \quad\left(C_{s-1}^{k}\right), \quad 0 \leqslant k \leqslant(s-1) .
$$

Кратности $C_{r}^{k}$ представляют собой биномиальные коэффициенты.

Характер неприводимого представления $(p, q)$ алгебры $A_{2}$ дается формулой Вейля

$$
\pi^{(p, q)}\left(\exp \left(\tau_{1} h_{1}+\tau_{2} h_{2}\right)\right)=\frac{\operatorname{Det}\left(\begin{array}{ccc}
e^{\tau_{1} l_{1}} & e^{\tau_{1} l_{2}} & e^{\tau_{1} l_{3}} \\
e^{\left(\tau_{2}-\tau_{1}\right) l_{1}} & e^{\left(\tau_{2}-\tau_{1}\right) l_{2}} & e^{\left(\tau_{2}-\tau_{1}\right) l_{3}} \\
e^{-\tau_{2} l_{1}} & e^{-\tau_{2} l_{2}} & e^{-\tau_{2} l_{3}}
\end{array}\right)}{\operatorname{Det}\left(\begin{array}{ccc}
e^{\tau_{1}} & 1 & e^{-\tau_{1}} \\
e^{\left(\tau_{2}-\tau_{1}\right)} & 1 & e^{-\left(\tau_{2}-\tau_{1}\right)} \\
e^{-\tau_{2}} & 1 & e^{\tau_{2}}
\end{array}\right)}
$$

где $l_{1}-l_{2}=p+1, l_{2}-l_{3}=q+1, l_{1}+l_{2}+l_{3}=0$. Вычисление определителей приводит к формуле

$$
\begin{aligned}
\pi^{(p, q)}= & \frac{1}{1-e^{-\left(\tau_{2}+\tau_{1}\right)}}\left[e^{\left(\tau_{2}-\tau_{1}\right)(p-q)}\left(e^{p x}+\cdots+1\right)\left(e^{q y}+\cdots+1\right)-\right. \\
& \left.-e^{-\tau_{1}(q+1)} e^{-\tau_{2}(p+1)}\left(e^{q x}+\cdots+1\right)\left(e^{p y}+\cdots+1\right)\right]
\end{aligned}
$$

где $x=2 \tau_{1}-\tau_{2}, y=2 \tau_{2}-\tau_{1}, x+y=\tau_{2}+\tau_{1}$. Очевидно, что при условиях $\tau_{2}+\tau_{1}=0$, $x+y=0$ последнее выражение переходит в сумму экспонент, аргументы которых представляют собой различные линейные комбинации $\tau$ с определенными коэффициентами. 
Редукция формулы (4.5) или (4.6) к подгруппам $A_{1}$ с инфинитезимальными генераторами $X^{+}=\left[X_{1}^{+}, X_{2}^{+}\right], \quad X^{-}=\left[X_{1}^{-}, X_{2}^{-}\right], \quad H=h_{1}+h_{2}$ эквивалентна подстановке $\tau_{1}=\tau_{2}=t$, что приводит к окончательному результату

$$
\begin{aligned}
\pi^{(p, q)}\left(\exp \left(h_{1}+h_{2}\right) t\right) & =e^{-(p+q) t} \frac{\sum_{k=0}^{p+q+1} e^{(p+q+1-k) t} \sum_{r=0}^{q} e^{(q-r) t} \sum_{s=0}^{p} e^{(p-s) t}}{\left(e^{t}+1\right)}= \\
& =\sum_{m=0}^{2(p+q)} e^{(p+q-m) t} c_{m}(p, q) .
\end{aligned}
$$

Редукция к подгруппам $A_{1}$, соответствующим генераторам матриц $X_{1}^{ \pm}, X_{2}^{ \pm}$первого и второго простых корней и генераторам матриц $h_{1}$ и $h_{2}$, приводит к таким же выражениям. Этот результат является очевидным и без всяких вычислений, поскольку элементы Картана всех корней алгебры $A_{2}$ связаны дискретными преобразованиями Вейля. До обшего рассмотрения мы приведем конкретный пример $(2,1)$-представления алгебры $A_{2}$. Этот пример отражает основные моменты всего построения.

4.1. (2, 1)-представление алгебры $A_{2}$. Прямое вычисление характера $(2,1)$-представления приводит (в соответствии с уравнением (4.6)) к сумме экспонент, которые мы записываем в определенном порядке, смысл которого скоро станет ясен:

$$
\begin{aligned}
\pi^{(2,1)}(\tau)= & e^{\left(2 \tau_{1}+\tau_{2}\right)}+e^{2 \tau_{2}}+e^{\left(3 \tau_{1}-\tau_{2}\right)}+e^{\left(-2 \tau_{1}+3 \tau_{2}\right)}+2 e^{\tau_{1}}+2 e^{\left(-\tau_{1}+\tau_{2}\right)}+e^{\left(2 \tau_{1}-2 \tau_{2}\right)}+ \\
& +e^{-\left(3 \tau_{1}-2 \tau_{2}\right)}+2 e^{-\tau_{2}}+e^{-2 \tau_{1}}+e^{\left(\tau_{1}-3 \tau_{2}\right)}+e^{-\left(\tau_{1}+2 \tau_{2}\right)}
\end{aligned}
$$

Рассмотрим редукцию этого выражения к $S L(2, R)$-подгруппе, связанной с составным корнем алгебры $A_{2}$. Записывая $\tau_{1}=\tau_{2}=t$, получаем

$$
\pi=e^{3 t}+2 e^{2 t}+3 e^{t}+3+3 e^{-t}+2 e^{-2 t}+e^{-3 t} .
$$

Поскольку генератор $h_{1}+h_{2}$ принимает равные единице значения на каждом из простых корней алгебры $A_{2}$, состояния старших (младших) векторов являются синглетными, а подпространства с двумя или пятью понижающими операторами - двумерными и т.д. Значения, принимаемые оператором $R_{1} R_{2} \equiv \exp \left[\left(h_{1}+h_{2}\right) t\right]$ на базисных векторах $(2,1)$-представления, в точности являются экспонентами из записанной выше суммы. Используя те же аргументы, мы находим значения, принимаемые генераторами $R_{1}$ и $R_{2}$ на этом базисе. Представим их в виде строк, придерживаясь необходимого порядка,

$$
\begin{aligned}
& R_{1}=\left(e^{2 t}, 1, e^{3 t}, e^{-2 t}, e^{t}, e^{t}, e^{-t}, e^{-t}, e^{2 t}, e^{-3 t}, 1,1, e^{-2 t}, e^{t}, e^{-t}\right), \\
& R_{2}=\left(e^{t}, e^{2 t}, e^{-t}, e^{3 t}, 1,1, e^{t}, e^{t}, e^{-2 t}, e^{2 t}, e^{-t}, e^{-t}, 1, e^{-3 t}, e^{-2 t}\right) .
\end{aligned}
$$

Данное представление определяет размеры матриц $\alpha$ и $\beta$ и явные выражения для них в диагональном виде. Матрищы $\alpha_{i}$ имеют следующий явный вид (скобки обозначают диагональные элементы):

$$
\begin{array}{llll}
\alpha_{1}=e^{2 t}, & \alpha_{2}=\left(1, e^{3 t}\right), & \alpha_{3}=\left(e^{-2 t} e^{t}, e^{t}\right), & \alpha_{4}=\left(e^{-t}, e^{-t}, e^{2 t}\right), \\
\alpha_{5}=\left(e^{-3 t}, 1,1\right), & \alpha_{6}=\left(e^{-2 t}, e^{t}\right), & \alpha_{7}=e^{-t} .
\end{array}
$$


Знание явных выражений для матриц $\alpha$ позволяет без труда разрешить первую строчку системы (3.5) и представить матрищу $r^{1}$ в виде

$$
r^{1}=\left(\begin{array}{ccccccccccccccc}
e^{2 t} & X & 0 & 0 & 0 & 0 & 0 & 0 & 0 & 0 & 0 & 0 & 0 & 0 & 0 \\
-X & 1 & 0 & A & 0 & 0 & 0 & 0 & 0 & 0 & 0 & 0 & 0 & 0 & 0 \\
0 & 0 & e^{3 t} & 0 & B & C & 0 & 0 & 0 & 0 & 0 & 0 & 0 & 0 & 0 \\
0 & -A & 0 & e^{-2 t} & 0 & 0 & 0 & 0 & 0 & 0 & 0 & 0 & 0 & 0 & 0 \\
0 & 0 & -B & 0 & e^{t} & 0 & D & E & 0 & 0 & 0 & 0 & 0 & 0 & 0 \\
0 & 0 & -C & 0 & 0 & e^{t} & F & G & 0 & 0 & 0 & 0 & 0 & 0 & 0 \\
0 & 0 & 0 & 0 & -D & -F & e^{-t} & 0 & 0 & H & 0 & 0 & 0 & 0 & 0 \\
0 & 0 & 0 & 0 & -E & -G & 0 & e^{-t} & 0 & K & 0 & 0 & 0 & 0 & 0 \\
0 & 0 & 0 & 0 & 0 & 0 & 0 & 0 & e^{2 t} & 0 & L & M & 0 & 0 & 0 \\
0 & 0 & 0 & 0 & 0 & 0 & -H & -K & 0 & e^{-3 t} & 0 & 0 & 0 & 0 & 0 \\
0 & 0 & 0 & 0 & 0 & 0 & 0 & 0 & -L & 0 & 1 & 0 & N & 0 & 0 \\
0 & 0 & 0 & 0 & 0 & 0 & 0 & 0 & -M & 0 & 0 & 1 & P & 0 & 0 \\
0 & 0 & 0 & 0 & 0 & 0 & 0 & 0 & 0 & 0 & -N & -P & e^{-2 t} & 0 & 0 \\
0 & 0 & 0 & 0 & 0 & 0 & 0 & 0 & 0 & 0 & 0 & 0 & 0 & e^{t} & Y \\
0 & 0 & 0 & 0 & 0 & 0 & 0 & 0 & 0 & 0 & 0 & 0 & 0 & -Y & e^{-t}
\end{array}\right) .
$$

Матрица $s^{1}$ получается из $r^{1}$ путем удаления диагональных элементов и такого изменения оставшейся матрицы, чтобы она стала симметричной, а не антисимметричной. Здесь мы предполагаем, что что $s^{1}$ можно выбрать в виде симметричной матрицы. По тем же причинам $r^{2}$ имеет вид

$$
r^{2}=\left(\begin{array}{ccccccccccccccc}
e^{t} & 0 & y & 0 & 0 & 0 & 0 & 0 & 0 & 0 & 0 & 0 & 0 & 0 & 0 \\
0 & e^{2 t} & 0 & 0 & n & p & 0 & 0 & 0 & 0 & 0 & 0 & 0 & 0 & 0 \\
-y & 0 & e^{-t} & 0 & 0 & 0 & 0 & 0 & 0 & 0 & 0 & 0 & 0 & 0 & 0 \\
0 & 0 & 0 & e^{3 t} & 0 & 0 & h & k & 0 & 0 & 0 & 0 & 0 & 0 & 0 \\
0 & -n & 0 & 0 & 1 & 0 & 0 & 0 & l & l & 0 & 0 & 0 & 0 & 0 \\
0 & -p & 0 & 0 & 0 & 1 & 0 & 0 & m & 0 & 0 & 0 & 0 & 0 & 0 \\
0 & 0 & 0 & -h & 0 & 0 & e^{t} & 0 & 0 & 0 & d & f & 0 & 0 & 0 \\
0 & 0 & 0 & -k & 0 & 0 & 0 & e^{t} & 0 & 0 & e & g & 0 & 0 & 0 \\
0 & 0 & 0 & 0 & -l & -m & 0 & 0 & e^{-2 t} & 0 & 0 & 0 & 0 & 0 & 0 \\
0 & 0 & 0 & 0 & 0 & 0 & 0 & 0 & 0 & e^{2 t} & 0 & 0 & a & 0 & 0 \\
0 & 0 & 0 & 0 & 0 & 0 & -d & -e & 0 & 0 & e^{-t} & 0 & 0 & b & 0 \\
0 & 0 & 0 & 0 & 0 & 0 & -f & -g & 0 & 0 & 0 & e^{-t} & 0 & c & 0 \\
0 & 0 & 0 & 0 & 0 & 0 & 0 & 0 & 0 & -a & 0 & 0 & 1 & 0 & x \\
0 & 0 & 0 & 0 & 0 & 0 & 0 & 0 & 0 & 0 & -b & -c & 0 & e^{-3 t} & 0 \\
0 & 0 & 0 & 0 & 0 & 0 & 0 & 0 & 0 & 0 & 0 & 0 & -x & 0 & e^{-2 t}
\end{array}\right) .
$$

Пока мы решили первые две строки уравнений системы (4.4). Теперь можно решить систему (4.4) в целом. Из первых строк второй части уравнений (4.4) получается (при $\left.a_{1,0}=b_{1,0}=0\right)$

$$
X^{2}=e^{t} \operatorname{sh} t \operatorname{sh}(2 t), \quad y^{2}=\operatorname{sh}^{2} t .
$$

Решение двух следуюших строк имеет вид

$$
\begin{array}{cc}
A^{2}=e^{-t} \operatorname{sh} t \operatorname{sh}(2 t), & B^{2}+C^{2}=e^{2 t} \operatorname{sh} t \operatorname{sh}(3 t), \\
n B+p C=e^{t} y X, & n^{2}+p^{2}=e^{t} \operatorname{sh} t \operatorname{sh}(2 t) .
\end{array}
$$

4 Теоретическая и математическая физика, т. 123, № 2, 2000 г. 
Эти уравнения определяют два двумерных вектора с заданным углом между ними:

$$
\begin{gathered}
(B, C)=e^{t}(\operatorname{sh} t \operatorname{sh}(3 t))^{\frac{1}{2}} t\left(\cos \phi_{1}, \sin \phi_{1}\right) \\
(n, p)=e^{\frac{t}{2}}(\operatorname{sh} t \operatorname{sh}(2 t))^{\frac{1}{2}}\left(\cos \phi_{2}, \sin \phi_{2}\right) \\
\cos \left(\phi_{1}-\phi_{2}\right)=\left(\frac{\operatorname{sh} t}{\operatorname{sh}(3 t)}\right)^{\frac{1}{2}}
\end{gathered}
$$

Ниже будет использована калибровка $\phi_{2}=0(p=0)$ (однако такой выбор калибровки абсолютно несушествен). На третьем шаге система уравнений принимает вид

$$
\begin{gathered}
D F+E G=e^{-2 t} B C=\operatorname{sh}^{2} t(2 \operatorname{ch}(2 t))^{\frac{1}{2}}, \\
D^{2}+E^{2}=e^{-2 t} B^{2}+\operatorname{sh}^{2} t=2 \operatorname{sh}^{2} t, \\
F^{2}+G^{2}=e^{-2 t} C^{2}+\operatorname{sh}^{2} t=\operatorname{sh} t \operatorname{sh}(3 t), \\
l^{2}=e^{-2 t} n^{2}, \quad m^{2}=e^{-2 t} p^{2}=0, \quad m l=e^{-2 t} n p=0, \\
h^{2}+k^{2}=e^{2 t} \operatorname{sh} t \operatorname{sh}(3 t), \quad m=0, \\
D h+E k=e^{t} A n=e^{t} \operatorname{sh} t \operatorname{sh}(2 t), \quad F h+G k=e^{t} A p=0 .
\end{gathered}
$$

Очевидно, что матрищы $s^{1}, s^{2}$ и $r^{1}, r^{2}$ инвариантны относительно двумерных врашений в плоскостях $(5,6),(7,8)$ и $(11,12)$ (в результате чего оказалось возможным выбрать $p=0$ на втором шаге вычислений с помощью поворотов в плоскости $(5,6))$. Теперь мы можем использовать эту инвариантность, выбирая в уравнениях на четвертом шаге $E=0$. Тогда все приведенные выше уравнения легко решаются. В результате получаем

$$
\begin{gathered}
D= \pm(2)^{\frac{1}{2}} \operatorname{sh} t, \quad F= \pm \operatorname{sh} t(\operatorname{ch}(2 t))^{\frac{1}{2}}, \quad G^{2}=\frac{1}{2} \operatorname{sh}^{2}(2 t) \\
l^{2}=e^{-t} \operatorname{sh} t \operatorname{sh}(2 t), \quad m=0, \quad h= \pm(2)^{-\frac{1}{2}} e^{t} \operatorname{sh}(2 t), \quad k= \pm e^{t} \operatorname{sh} t(\operatorname{ch}(2 t))^{\frac{1}{2}}
\end{gathered}
$$

На пятом шаге используем в полученных уравнениях $(11,12)$-инвариантность, чтобы фиксировать $M=0$. Учитывая это, получаем

$$
\begin{gathered}
H K=e^{-2 t} F G= \pm 2^{-\frac{1}{2}} \operatorname{sh} t \operatorname{sh}(2 t)(\operatorname{ch}(2 t))^{\frac{1}{2}} \\
H^{2}=e^{-2 t}\left(D^{2}+F^{2}-\operatorname{sh}^{2} t\right)=\frac{1}{2} e^{-2 t} \operatorname{sh}^{2}(2 t) \\
K^{2}=e^{-2 t}\left(G^{2}-\operatorname{sh}^{2} t\right)=e^{-2 t} \operatorname{sh}^{2} t \operatorname{ch}(2 t), \\
L^{2}=e^{2} \operatorname{sh} t \operatorname{sh}(2 t), \quad 2 t d L+f M=e^{t}(D l+F m), \\
e L+g M=e^{t}(E l+G m), \quad d=D, \quad e=E=0 \\
e^{2}+g^{2}=e^{-2 t} k^{2}+\operatorname{sh}^{2} t=\frac{1}{2} \operatorname{sh}^{2}(2 t), \\
d e+f g=e^{-2 t} h k=2^{-\frac{1}{2}} \operatorname{sh} t \operatorname{sh}(2 t)(\operatorname{ch}(2 t))^{\frac{1}{2}} \\
d^{2}+f^{2}=e^{-2 t} h^{2}+\operatorname{sh}^{2} t=\operatorname{sh}^{2} t(\operatorname{ch}(2 t)+2), \quad l^{2}=e^{-t} \operatorname{sh} t \operatorname{sh}(2 t)
\end{gathered}
$$


Решение этой системы элементарно. Приведем окончательный результат:

$$
d=D, \quad e=E=0, \quad f=F, \quad g=G .
$$

Явньй вид $H$ и $K$ дан выше. Мы не будем воспроизводить здесь два оставшихся шага вычислений, потому что они просты и не содержат ничего нового.

Отметим, что на каждом последуюшем шаге мы всегда имеем систему квадратных уравнений только для неизвестных матричных элементов матриц $a_{n+1, n}, a_{n, n+1}, b_{n+1, n}$ и $b_{n, n+1}$, правая часть которых была получена на предыдушем шаге. Вследствие условий инвариантности число уравнений точно равно числу неизвестных переменных. Caмосогласованность всей конструкции есть только следствие глобальной теории представлений.

4.2. Общий случай $(p, q)$-представления. Чтобы обобщить результаты раздела 4.1 на случай произвольного $(p, q)$-представления алгебры $A_{2}$, необходима более подробная информация о структуре матриц $a_{n, n \pm 1}$ и $b_{n, n \pm 1}$. Первые две строки системы (4.4) накладывают на них дополнительные ограничения. А именно отличными от нуля являются только те матричные элементы $\left(a_{n, n \pm 1}\right)_{s, s^{\prime}}$ и $\left(b_{n, n \pm 1}\right)_{s, s^{\prime}}$, для которых $\left(s, s^{\prime}\right)$ удовлетворяют условиям

$$
\frac{\alpha_{n}^{s}}{\alpha_{n \pm 1}^{s^{\prime}}}=e^{ \pm 2 t}, \quad \frac{\beta_{n}^{s}}{\beta_{n \pm 1}^{s^{\prime}}}=e^{ \pm 2 t}
$$

В свою очередь, диагональные элементы матриц $\alpha$ и $\beta$ связаны соотношениями

$$
\frac{\alpha_{n}^{s}}{\alpha_{n}^{s+1}}=e^{-3 t}, \quad \frac{\beta_{n}^{s}}{\beta_{n}^{s+1}}=e^{3 t}
$$

Обозначим кратности диагональных элементов $\alpha_{n}^{s}$ и $\beta_{n}^{s}$ через $N_{n}^{s}$. Очевидно, что $N_{n}=$ $\sum_{s} N_{n}^{s}$. Кратность $N_{n}^{s}$ в точности равна целому числу в сответствуюшей экспоненте в формуле Вейля для характеров $\left(1.1^{\prime}\right)$. После редукции к $A_{1}$-подгруппе, определяемой первым (вторым) простым корнем, она в точности равна коэффицциенту при $\alpha_{n}^{s}$ (соответственно $\left.\beta_{n}^{s}\right)$.

Каждая матрица $a_{n, n+1}$ (для определенности выберем знак плюс) разбивается на прямоугольные блочные $\left(N_{n}^{s} \times N_{n+1}^{s^{\prime}}\right)$-матрицы $a_{n, n+1}^{s, s^{\prime}}$, где $\left(s, s^{\prime}\right)$ - индексы диагональных матричных элементов $\alpha_{n}^{s}$ и $\alpha_{n+1}^{s^{\prime}}$ соответственно. Отличны от нуля только те блочные матрицы, для которых $\alpha_{n}^{s} / \alpha_{n+1}^{s^{\prime}}=e^{ \pm 2 t}$. Это обстоятельство имеет два важных следствия. Во-первых, в строке (с “широким" $N_{n}^{s}$ ) и в столбце (с “широким" $N_{n+1}^{s^{\prime}}$ ) может быть только одна отличная от нуля блочная матрица. Во-вторых, все матрицы $a_{n, n+1}^{s \pm k, s^{\prime} \pm k}$ с индексами, сдвинутыми на целое положительное число $k$, отличны от нуля одновременно с $a_{n, n+1}^{s, s^{\prime}}$ (конечно, при условии, что индексы $s \pm k, s^{\prime} \pm k$ находятся внутри области их определения). 
Из явного вида анзаца для генераторов $s^{1}, s^{2}$ и $r^{1}, r^{2}$ в начале данного раздела следует их форм-инвариантность при всех не меняюших диагональные матричные элементы канонических $S L\left(N_{n}^{s} ; R\right)$-преобразованиях, изменяющих матрицы $a$ по закону

$$
a \rightarrow G\left(N_{n}^{s}, R\right) a G\left(N_{n+1}^{s^{\prime}}, R\right)
$$

Если мы хотим сохранить симметрию матриц $s^{1}$ и $s^{2}$ (до настояшего моменты мы считали их симметричными), необходимо редуцировать это преобразование до прямого произведения ортогональных $O_{N_{n}^{s}}$-преобразований. Поскольку к матрицам $b$ применимы те же соображения, мы можем переписать остаюшиеся уравнения системы (4.4) (три последние строки) в терминах только “примитивных" блочных матрищ:

$$
\begin{gathered}
e^{-t} a_{n, n-1}^{s, s+2} a_{n-1, n}^{s+2, s}-e^{t} a_{n, n+1}^{s, s-2} a_{n+1, n}^{s-2, s}=\alpha_{n}^{s} \operatorname{sh} t \operatorname{sh}\left(\ln \alpha_{n}^{s}\right) I_{N_{n}^{s}}, \\
e^{-\frac{t}{2}} b_{n, n+1}^{s, s+1} a_{n+1, n}^{s+1, s+3}=e^{\frac{t}{2}} a_{n, n-1}^{s, s+2} b_{n-1, n}^{s+2, s+3}, \\
e^{-t} b_{n, n-1}^{s, s-1} b_{n-1, n}^{s-1, s}-e^{t} b_{n, n+1}^{s, s+1} b_{n+1, n}^{s+1, s}=-\operatorname{sh} t\left(\lambda_{n}\left(\alpha_{n}^{s}\right)^{-1}\right) \operatorname{sh}\left(\ln \left(\lambda_{n}\left(\alpha_{n}^{s}\right)^{-1}\right)\right) I_{N_{n}^{s}},
\end{gathered}
$$

где $I_{N_{n}^{s}}$ - единичная $\left(N_{n}^{s} \times N_{n}^{s}\right)$-матрица и индекс $s$ пробегает все возможные значения. Исходная система (4.4), таким образом, распадается в систему уравнений типа цепочки для блочных матриц, и каждая цепочка остается абсолютно не зависимой от других. На каждом шаге вычислений требуется найти прямоугольные матришы $a$ и $b$ заданного размера, удовлетворяющие системе алгебраических уравнений

$$
a a^{T}=A, \quad b b^{T}=B, \quad b a^{T}=C, \quad a b^{T}=C^{T},
$$

где $A, B$ и $C$ - известные матрицы. В соответствии с (4.8) они определены на предыдушем шаге вычислений (в точности из системы (4.9)) вместе с $\alpha_{n}^{s}$ и $\beta_{n}^{s}$, известными из формулы Вейля, что гарантирует согласованность всего построения.

Проиллюстрируем проведенное выше рассмотрение на примере из предыдушего раздела. Анзацы для $s^{1}, s^{2}$ и $r^{1}, r^{2}$ имеют блочную структуру

$$
\begin{gathered}
a_{1,2}^{2,0}=X, \quad a_{2,3}^{0,-2}=A, \quad a_{2,3}^{3,1}=\left(\begin{array}{ll}
B & C
\end{array}\right), \quad a_{3,4}^{1,-1}=\left(\begin{array}{cc}
D & E \\
F & G
\end{array}\right), \\
a_{4,5}^{-1,-3}=\left(\begin{array}{c}
H \\
K
\end{array}\right), \quad a_{4,5}^{2,0}=\left(\begin{array}{ll}
L & M
\end{array}\right), \quad a_{5,6}^{0,-2}=\left(\begin{array}{c}
N \\
P
\end{array}\right), \quad a_{6,7}^{1,-1}=Y, \\
b_{1,2}^{2,3}=y, \quad b_{2,3}^{0,1}=\left(\begin{array}{ll}
n & p
\end{array}\right), \quad b_{3,4}^{-2,-1}=\left(\begin{array}{ll}
h & k
\end{array}\right), \quad b_{3,4}^{1,2}=\left(\begin{array}{c}
l \\
m
\end{array}\right), \\
b_{4,5}^{-1,0}=\left(\begin{array}{cc}
d & f \\
e & g
\end{array}\right), \quad b_{5,6}^{0,1}=\left(\begin{array}{c}
b \\
c
\end{array}\right), \quad b_{5,6}^{-3,-2}=a, \quad b_{6,7}^{-2,-1}=x .
\end{gathered}
$$


Продемонстрируем структуру типа цепочки на примере уравнений, связывающих примитивные матрицы $a$ :

$$
\begin{gathered}
-e^{t} a_{1,2}^{2,0} a_{2,1}^{0,2}=-e^{2 t} \operatorname{sh} t \operatorname{sh}(2 t), \quad e^{-t} a_{2,1}^{0,2} a_{1,2}^{2,0}-e^{t} a_{2,3}^{0,-2} a_{3,2}^{-2,0}=0 \\
e^{-t} a_{3,2}^{-2,0} a_{2,3}^{0,-2}=e^{-2 t} \operatorname{sh} t \operatorname{sh}(2 t), \quad-e^{t} a_{2,3}^{3,1} a_{3,2}^{1,3}=-e^{3 t} \operatorname{sh} t \operatorname{sh}(3 t), \\
e^{-t} a_{3,2}^{1,3} a_{2,3}^{3,1}-e^{t} a_{3,4}^{1,-1} a_{4,3}^{-1,1}=-e^{t} \operatorname{sh}^{2} t \\
e^{-t} a_{4,3}^{-1,1} a_{3,4}^{1,-1}-e^{t} a_{4,5}^{-1,-3} a_{5,4}^{-3,-1}=-e^{t} \operatorname{sh}^{2} t \\
e^{-t} a_{5,4}^{-3,-1} a_{4,5}^{-1,-3}=e^{-3 t} \operatorname{sh}^{2} t, \quad-e^{t} a_{4,5}^{2,0} a_{5,4}^{0,2}=-e^{2 t} \operatorname{sh} t \operatorname{sh}(2 t) \\
e^{-t} a_{5,4}^{0,2} a_{4,5}^{2,0}-e^{t} a_{5,6}^{0,-2} a_{5,6}^{-2,0}=0, \quad e^{t} a_{5,6}^{-2,0} a_{6,5}^{0,-2}=e^{2 t} \operatorname{sh} t \operatorname{sh}(2 t) \\
e^{t} a_{6,7}^{1,-1} a_{7,6}^{-1,1}=e^{t} \operatorname{sh}^{2} t
\end{gathered}
$$

Здесь мы имеем две цепочки с тремя элементами (спина 1), одну цепочку с четырьмя элементами (спина $3 / 2$ ) и одну скалярную цепочку.

\section{5. АЛГЕБРА $B_{2}$}

В этом случае $p=2$. Мы опять используем представление, где оператор $Q$ имеет диагональный вид. Из уравнения (3.7) следует, что его диагональные матричные элементы связаны условием $\lambda_{i} / \lambda_{i+1}=e^{2 t}$. Кроме того, генераторы $Q_{1}^{ \pm}$коммутируют с $Q$, следовательно, $s^{1}$ и $r^{1}$ имеют блочный вид

$$
s^{1}=\left(\begin{array}{lll}
\ldots & a_{i} & \ldots
\end{array}\right), \quad r^{1}=\left(\begin{array}{lll}
\ldots & b_{i} & \ldots
\end{array}\right)
$$

a $s^{2}$ и $r^{2}$ сохраняют свой прежний вид

$$
s^{2}=\left(\begin{array}{lllll}
\ldots & a_{i, i-1} & 0 & a_{i, i+1} & \ldots
\end{array}\right), \quad r^{2}=\left(\begin{array}{lllll}
\ldots & -a_{i, i-1} & \alpha_{i} & a_{i, i+1} & \ldots
\end{array}\right) .
$$

Первое из уравнений (3.1) сохраняет свой вид для блоков матриц $\left(s^{1}, r^{1}\right)$ :

$$
\left[a_{i}, b_{i}\right]=\operatorname{th} t\left(a_{i}^{2}-b_{i}^{2}+1\right) .
$$

Второе из уравнений (3.1) эквивалентно уравнению (4.1) с заменой $t \rightarrow 2 t$ :

$$
\begin{gathered}
e^{-2 t} a_{n, n-1} \alpha_{n-1}=e^{2 t} \alpha_{n} a_{n, n-1}, \quad e^{2 t} a_{n, n+1} \alpha_{n+1}=e^{-2 t} \alpha_{n} a_{n, n+1}, \\
2 e^{-2 t} a_{n, n-1} a_{n-1, n}-2 e^{2 t} a_{n, n+1} a_{n+1, n}=\operatorname{sh} t\left(I_{n}-\alpha_{n}^{2}\right) .
\end{gathered}
$$

Система уравнений имеет вид

$$
\begin{aligned}
& e^{-t}\left(a_{i}+b_{i}\right) a_{i, i-1}=e^{t} a_{i, i-1}\left(a_{i-1}+b_{i-1}\right), \\
& e^{t}\left(a_{i}-b_{i}\right) a_{i, i+1}=e^{-t} a_{i, i+1}\left(a_{i+1}-b_{i+1}\right), \\
& e^{t} \alpha_{i}\left(a_{i}+b_{i}\right)-e^{-t}\left(a_{i}+b_{i}\right) \alpha_{i}=2 \operatorname{sh} t \lambda_{i} I_{i}, \\
& e^{-t} \alpha_{i}\left(a_{i}-b_{i}\right)-e^{t}\left(a_{i}-b_{i}\right) \alpha_{i}=2 \operatorname{sh} t \lambda_{i} I_{i} .
\end{aligned}
$$


Группа Вейля алгебры $B_{2}$ состоит из восьми элементов. Характер ее $(p, q)$-представления, вычисленный с помошью $\left(1.1^{\prime}\right)$, записывается как

$$
\pi^{(p, q)}\left(\tau_{1}, \tau_{2}\right)=\frac{\operatorname{sh}\left(l_{1} \tilde{\tau}_{2}\right) \operatorname{sh}\left(l_{2} \tilde{\tau}_{2}\right)-\operatorname{sh}\left(l_{1} \tilde{\tau}_{2}\right) \operatorname{sh}\left(l_{2} \tilde{\tau}_{1}\right)}{\operatorname{sh}\left(2 \tilde{\tau}_{1}\right) \operatorname{sh}\left(\tilde{\tau}_{2}\right)-\operatorname{sh}\left(\tilde{\tau}_{1}\right) \operatorname{sh}\left(2 \tilde{\tau}_{2}\right)},
$$

где $l_{1}-l_{2}=p+1, l_{2}=q+1, l_{1}=p+q+2, l_{1}+l_{2}=p+q+3, \tilde{\tau}_{1}=\tau_{1}, \tilde{\tau}_{2}=\tau_{2}-\tau_{1}$.

Все диагональные элементы матриц $R_{1}, R_{2}$ и $Q=R_{1} R_{2}$, необходимые для дальнейших вычислений, следует получить из уравнений (5.4).

Теперь подробно рассмотрим конкретный случай 16 -мерного $(1,1)$-представления алгебры $B_{2}$. В этом случае $l_{1}=4$ и $l_{2}=2$, а характер $(1,1)$-представления в соответствии с уравнением (5.4) имеет вид

$$
\begin{aligned}
\pi^{(1,1)}\left(\tau_{1}, \tau_{2}\right)= & \left(e^{\tilde{\tau}_{1}}+e^{-\tilde{\tau}_{1}}\right)\left(e^{\tilde{\tau}_{2}}+e^{-\tilde{\tau}_{2}}\right)\left(e^{\tilde{\tau}_{1}}+e^{\tilde{\tau}_{2}}\right)\left(1+e^{-\left(\tilde{\tau}_{1}+\tilde{\tau}_{2}\right)}\right)= \\
= & e^{2 \tau_{2}-\tau_{1}}+e^{\tau_{2}+\tau_{1}}+e^{2 \tau_{2}-3 \tau_{1}}+2 e^{\tau_{2}-\tau_{1}}+2 e^{\tau_{1}}+e^{-\tau_{2}+3 \tau_{1}}+ \\
& +e^{\tau_{2}-3 \tau_{1}}+2 e^{-\tau_{1}}+2 e^{-\tau_{2}+\tau_{1}}+e^{-2 \tau_{2}+3 \tau_{1}}+e^{-\tau_{2}-\tau_{1}}+e^{-2 \tau_{2}+\tau_{1}} .
\end{aligned}
$$

После приведения к $A_{1}$-подгруппе, соответствуюшей генератору первого сложного корня алгебры $B_{2}\left(X_{1,2}^{ \pm}, H=h_{1}+2 h_{2}\right)$, что эквивалентно подстановке $\tau_{1}=t, \tau_{2}=2 t$ в выражение для характера, получаем

$$
\pi^{(1,1)}(t, 2 t)=2 e^{3 t}+6 e^{t}+6 e^{-t}+2 e^{-3 t} .
$$

Приведение к $A_{1}$-подгруппе, соответствуюшей генератору первого простого корня ( $X_{1}^{ \pm}$, $H=h_{1}, \tau_{1}=t, \tau_{2}=0$ ), дает

$$
\pi^{(1,1)}(t, 0)=e^{-t}+e^{t}+e^{-3 t}+2 e^{-t}+2 e^{t}+e^{3 t}+e^{-3 t}+2 e^{-t}+2 e^{t}+e^{3 t}+e^{-t}+e^{t} .
$$

Таким образом, получаем матрицы $\alpha$ в явном виде:

$$
\begin{gathered}
\alpha_{1}=\left(e^{4 t}, e^{2 t}\right), \quad \alpha_{2}=\left(e^{4 t}, e^{2 t}, e^{2 t}, 1,1, e^{-2 t}\right), \\
\alpha_{3}=\left(e^{2 t}, 1,1, e^{-2 t}, e^{-2 t}, e^{-4 t}\right), \quad \alpha_{4}=\left(e^{-2 t}, e^{-4 t}\right)
\end{gathered}
$$

(как и раньше, в скобках заключены диагональные элементы матриц $\alpha$ ).

Прямоугольные матрицы из анзацев для $\left(s^{2}, r^{2}\right)$ имеют размеры

$$
a_{1,2} \rightarrow 2 \times 6, \quad a_{2,3} \rightarrow 6 \times 6, \quad a_{3,4} \rightarrow 6 \times 2,
$$

а размерности матрищ $a_{i}$ и $b_{i}$ из анзацев для $\left(s^{1}, r^{1}\right)$ равны

$$
a_{1}, b_{1} \rightarrow 2 \times 2, \quad a_{2}, b_{2} \rightarrow 6 \times 6, \quad a_{3}, b_{3} \rightarrow 6 \times 6, \quad a_{4}, b_{4} \rightarrow 2 \times 2 .
$$


Первая строка уравнений системы (5.2) имеет решение

$$
a_{1,2}=\left(\begin{array}{cccccc}
0 & 0 & 0 & A & B & 0 \\
0 & 0 & 0 & 0 & 0 & C
\end{array}\right), \quad a_{2,3}=\left(\begin{array}{cccccc}
0 & D & E & 0 & 0 & 0 \\
0 & 0 & 0 & F & G & 0 \\
0 & 0 & 0 & H & K & 0 \\
0 & 0 & 0 & 0 & 0 & L \\
0 & 0 & 0 & 0 & 0 & M \\
0 & 0 & 0 & 0 & 0 & 0
\end{array}\right), \quad a_{3,4}=\left(\begin{array}{cc}
N & 0 \\
0 & P \\
0 & R \\
0 & 0 \\
0 & 0 \\
0 & 0
\end{array}\right)
$$

или в обозначениях с использованием примитивных прямоугольных матрищ, введенных в предыдушем разделе,

$$
\begin{gathered}
a_{1,2}^{2,-2}=C, \quad a_{1,2}^{4,0}=\left(\begin{array}{cc}
A & B
\end{array}\right), \quad a_{2,3}^{4,0}=\left(\begin{array}{ll}
D & E
\end{array}\right), \quad a_{2,3}^{2,-2}=\left(\begin{array}{cc}
F & G \\
H & K
\end{array}\right), \\
a_{2,3}^{0,-4}=\left(\begin{array}{c}
L \\
M
\end{array}\right), \quad a_{3,4}^{0,-4}=\left(\begin{array}{c}
P \\
R
\end{array}\right), \quad a_{3,4}^{2,-2}=N .
\end{gathered}
$$

Отметим, что обозначения с использованием примитивных прямоугольных матрищ не зависят от выбора порядка экспонент в формуле Вейля для характеров.

Вторая строка системы (5.2) записывается как

$$
\begin{aligned}
& -e^{2 t} a_{1,2}^{2,-2} a_{2,1}^{-2,2}=-e^{2 t} \operatorname{sh}^{2}(2 t), \quad-e^{2 t} a_{1,2}^{4,0} a_{2,1}^{0,4}=-e^{4 t} \operatorname{sh}(2 t) \operatorname{sh}(4 t), \\
& e^{-2 t} a_{2,1}^{-2,2} a_{1,2}^{2,-2}=e^{-2 t} \operatorname{sh}^{2}(2 t), \quad e^{-2 t} a_{2,1}^{0,4} a_{1,2}^{4,0}-e^{2 t} a_{2,3}^{0,-4} a_{3,2}^{-4,0}=0, \\
& -e^{2 t} a_{2,3}^{2,-2} a_{3,2}^{-2,2}=-e^{2 t} \operatorname{sh}^{2}(2 t) I_{2}, \quad e^{-2 t} a_{3,2}^{-4,0} a_{2,3}^{0,-4}=e^{-4 t} \operatorname{sh}(2 t) \operatorname{sh}(4 t), \\
& e^{-2 t} a_{3,2}^{-2,2} a_{2,3}^{2,-2}=e^{-2 t} \operatorname{sh}^{2}(2 t) I_{2}, \quad e^{-2 t} a_{3,2}^{0,4} a_{2,3}^{4,0}-e^{2 t} a_{3,4}^{0,-4} a_{4,3}^{-4,0}=0, \\
& e^{-2 t} a_{4,3}^{-4,0} a_{3,4}^{0,-4}=e^{-4 t} \operatorname{sh}(2 t) \operatorname{sh}(4 t), \quad e^{-2 t} a_{4,3}^{-2,2} a_{3,4}^{2,-2}=e^{-2 t} \operatorname{sh}^{2}(2 t) .
\end{aligned}
$$

Хотя не все эти уравнения различны, здесь нет противоречия. Это может служить дополнительным аргументом в пользу самосогласованности всего построения. Мы приведем только неповторяющиеся уравнения:

$$
\begin{gathered}
C^{2}=\operatorname{sh}^{2}(2 t), \quad A^{2}+B^{2}=e^{2 t} \operatorname{sh}(2 t) \operatorname{sh}(4 t), \quad L= \pm e^{-2 t} A, \quad M= \pm e^{-2 t} B, \\
\left(\begin{array}{cc}
F & G \\
H & K
\end{array}\right)=\operatorname{sh}(2 t)\left(\begin{array}{cc}
\cos \phi & \sin \phi \\
-\sin \phi & \cos \phi
\end{array}\right), \\
D= \pm e^{2 t} P, \quad E= \pm e^{2 t} R, \quad P^{2}+R^{2}=e^{-2 t} \operatorname{sh}(2 t) \operatorname{sh}(4 t), \quad N^{2}=\operatorname{sh}^{2}(2 t),
\end{gathered}
$$

решения которых определены единственным образом с точностью до ортогональных преобразований. 
Перейдем теперь к уравнениям второй строки системы (5.3), связываюшим матрицы $\alpha$ и $a \pm b$. Прямые вычисления дают

$$
\begin{aligned}
& a_{1}+b_{1}=\left(\begin{array}{cc}
e^{-t} & 0 \\
x & e^{t}
\end{array}\right), \quad a_{1}-b_{1}=\left(\begin{array}{cc}
-e^{-t} & y \\
0 & -e^{t}
\end{array}\right), \\
& a_{4}+b_{4}=\left(\begin{array}{cc}
e^{-t} & 0 \\
u & e^{t}
\end{array}\right), \quad a_{4}-b_{4}=\left(\begin{array}{cc}
-e^{-t} & v \\
0 & -e^{t}
\end{array}\right), \\
& a_{2}+b_{2}=\left(\begin{array}{cccc}
e^{-3 t} & 0 & 0 & 0 \\
X_{2,1} & e^{-t} & 0 & 0 \\
0 & X_{3,2} & e^{t} & 0 \\
0 & 0 & X_{4,3} & e^{3 t}
\end{array}\right), \quad a_{2}-b_{2}=\left(\begin{array}{cccc}
-e^{-3 t} & Y_{1,2} & 0 & 0 \\
0 & -e^{-t} & Y_{2,3} & 0 \\
0 & 0 & -e^{t} & Y_{3,4} \\
0 & 0 & 0 & -e^{3 t}
\end{array}\right) \\
& a_{3}+b_{3}=\left(\begin{array}{cccc}
e^{-3 t} & 0 & 0 & 0 \\
U_{2,1} & e^{-t} & 0 & 0 \\
0 & U_{3,2} & e^{t} & 0 \\
0 & 0 & U_{4,3} & e^{3 t}
\end{array}\right), \quad a_{3}-b_{3}=\left(\begin{array}{cccc}
-e^{-3 t} & V_{1,2} & 0 & 0 \\
0 & -e^{-t} & V_{2,3} & 0 \\
0 & 0 & -e^{t} & V_{3,4} \\
0 & 0 & 0 & -e^{3 t}
\end{array}\right)
\end{aligned}
$$

$(6 \times 6)$-матрицы $a_{2} \pm b_{2}$ и $a_{3} \pm b_{3}$ представлены в четырехмерном виде с учетом того, что индексы 2,3 в действительности являются двумерными. Таким образом, $X_{21}$ и $Y_{12}$ представляют собой соответственно двумерные векторы $2 \times 1$ (столбцы) и $1 \times 2$ (строки) и т.д.

Теперь мы должны принять во внимание, что матрицы $a$ и $b$ удовлетворяют уравнениям (5.1). В терминах ( $a \pm b)$-матрищ последние уравнения можно переписать в виде

$$
e^{t}(a+b)(b-a)-e^{-t}(b-a)(a+b)=2 \operatorname{sh} t I .
$$

Прямая подстановка полученных выше выражений для $\left(a_{1}, b_{1}\right)$ и $\left(a_{4}, b_{4}\right)$ в последние уравнения приводит к следующему ограничению на параметры:

$$
x y=4 \operatorname{sh}^{2} t, \quad u v=4 \operatorname{sh}^{2} t .
$$

Если мы хотим иметь симметричную матрицу $s^{1}$, то необходимо, чтобы

$$
x=y=u=v=2 \operatorname{sh} t .
$$

Такой результат можно понять без всяких вычислений, рассматривая только соотношения

$$
\frac{a+b}{2 \operatorname{sh} t}=Q^{+}, \quad \frac{a-b}{2 \operatorname{sh} t}=Q^{-}
$$

из раздела 3 . Выражения для $\left(a_{1,4} \pm b_{1,4}\right)$ совпадают с выражениями для (двумерного) спинорного представления квантовой $A_{1}^{q}$-алгебры после дополнительного преобразования подобия с двумерной матрицей

$$
\sigma=\left(\begin{array}{ll}
0 & 1 \\
1 & 0
\end{array}\right)
$$


Такова же и ситуация с шестимерными матрицами. Необходимо рассмотреть прямую сумму четырехмерного (спина $3 / 2$ ) представления алгебры $A_{1}^{q}$, связанного с индексами $1,2,5,6$, и двумерного (спина $1 / 2$ ) представления, связанного с индексами 3,4 . После этого выполняется преобразование подобия с $(6 \times 6)$-матрицей с ненулевыми элементами на главной антидиагонали. В результате получаем в точности матрицу $a_{2,3} \pm b_{2,3}$. При такой процедуре возникают ограничения на параметры $X, Y, U$ и $V$ :

$$
\begin{gathered}
X_{2,1}=U_{2,1}=\left(\begin{array}{c}
2 e^{-t}(\operatorname{sh}(3 t) \operatorname{sh} t)^{\frac{1}{2}} \\
0
\end{array}\right), \quad X_{2,3}=U_{2,3}=\left(\begin{array}{cc}
0 & 2 \operatorname{sh} t \\
2 \operatorname{sh}(2 t) & 0
\end{array}\right) \\
X_{3,4}=U_{3,4}=\left(\begin{array}{ll}
0 & 2 e^{t}(\operatorname{sh}(3 t) \operatorname{sh} t)^{\frac{1}{2}}
\end{array}\right)
\end{gathered}
$$

а также аналогичные выражения для элементов $Y$ и $V\left(Y=X^{T}, V=U^{T}\right)$. Необходимо подчеркнуть, что мы фиксировали калибровку и поэтому все приведенные выше выражения не содержат дополнительных параметров. Это было достигнуто путем определенного выбора вида матриц $x, y, X, Y, u, v, U$ и $V$.

Наконец, нужно удовлетворить первой строке уравнений системы (5.3), связывающих прямоугольные матрищы $a_{n, n+1}$ с матрицами $a_{i} \pm b_{i}$. Прямые вычисления дают

$$
\begin{aligned}
e^{-t}\left(X_{4,3} a_{2,1}^{0,4}\right) & =e^{t} C x, \quad e^{-t}\left(U_{3,2} a_{2,3}^{4,0}\right)=e^{t}\left(a_{2,3}^{2,-2} X_{2,1}\right), \\
e^{-t}\left(U_{4,3} a_{2,3}^{2,-2}\right) & =e^{t}\left(a_{3,2}^{-4,0} X_{3,2}\right), \quad e^{-t} u N=e^{t}\left(a_{4,3}^{-4,0} U_{2,1}\right) .
\end{aligned}
$$

Все параметры в конструкции однозначно определены формулами

$$
\begin{gathered}
A=E=e^{t} \operatorname{sh}(2 t)\left(\frac{\operatorname{sh}(5 t)}{\operatorname{sh}(3 t)}\right)^{\frac{1}{2}}, \quad B=D=e^{t} \operatorname{sh}(2 t)\left(\frac{\operatorname{sh} t}{\operatorname{sh}(3 t)}\right)^{\frac{1}{2}}, \\
\sin \phi=-\frac{\operatorname{sh}(2 t)}{\operatorname{sh}(3 t)}, \quad \cos \phi=\frac{(\operatorname{sh}(5 t) \operatorname{sh} t)^{\frac{1}{2}}}{\operatorname{sh}(3 t)} .
\end{gathered}
$$

\section{6. АЛГЕБРА $G_{2}$}

Повторяя аргументы двух предыдущих разделов, запишем анзацы для матриц $\left(s^{1}, r^{1}\right)$ и $\left(s^{2}, r^{2}\right)$ в символической строчной форме:

$$
\begin{aligned}
s^{1} & =\left(\begin{array}{lllllll}
\ldots & a_{i, i-1} & 0 & a_{i, i+1} & \ldots
\end{array}\right) \\
r^{1} & =\left(\begin{array}{lllllllll}
\ldots & a_{i, i-1} & \alpha_{i} & -a_{i, i+1} & \ldots
\end{array}\right) \\
s^{2} & =\left(\begin{array}{lllllllll}
\ldots & b_{i, i-3} & 0 & 0 & 0 & 0 & 0 & b_{i, i+3} & \ldots
\end{array}\right) \\
r^{2} & =\left(\begin{array}{lllllllll}
\ldots & -b_{i, i-3} & 0 & 0 & \beta_{i} & 0 & 0 & a_{i, i+1} & \ldots
\end{array}\right) .
\end{aligned}
$$

Появление здесь $a_{i, i+1}$ вместо $a_{i, i-1}$, как в случае алгебры $A_{2}$, связано с различными знаками в первом из уравнений (3.5). В этой формуле сохранены обозначения предыдущих разделов: $\alpha_{i}$ и $\beta_{i}-$ квадратные $\left(N_{i} \times N_{i}\right)$-матрицы, $a_{p, q}$ и $b_{p, q}-$ прямоугольные $\left(N_{p} \times N_{q}\right)$-матрицы. Уравнение, связывающее $s^{1}$ и $r^{1}$, эквивалентно матричной системе

$$
\begin{gathered}
e^{t} a_{n, n-1} \alpha_{n-1}=e^{-t} \alpha_{n} a_{n, n-1}, \quad e^{-t} a_{n, n+1} \alpha_{n+1}=e^{t} \alpha_{n} a_{n, n+1}, \\
-2 e^{t} a_{n, n-1} a_{n-1, n}+2 e^{-t} a_{n, n+1} a_{n+1, n}=\operatorname{sh} t\left(I_{n}-\alpha_{n}^{2}\right),
\end{gathered}
$$


а из уравнений для $s^{2}$ и $r^{2}$ получаем систему

$$
\begin{gathered}
e^{-3 t} b_{n, n-3} \beta_{n-3}=e^{3 t} \beta_{n} b_{n, n-3}, \quad e^{3 t} b_{n, n+3} \beta_{n+3}=e^{-3 t} \beta_{n} b_{n, n+3}, \\
2 e^{-3 t} b_{n, n-3} b_{n-3, n}-2 e^{3 t} b_{n, n+3} b_{n+3, n}=\operatorname{sh} t\left(I_{n}-\beta_{n}^{2}\right),
\end{gathered}
$$

где $I_{n}$ - единичная $\left(N_{n} \times N_{n}\right)$-матрица. Из последнего из уравнений $(3.1)$ и определения $Q(3.2)$ следует система для смешанных матриц $a, \alpha$ и $b, \beta$ :

$$
\begin{gathered}
e^{\frac{3 t}{2}} b_{n, n-3} \alpha_{n-3}=e^{-\frac{3 t}{2}} \alpha_{n} b_{n, n-3}, \quad e^{\frac{3 t}{2}} a_{n, n+1} \beta_{n+1}=e^{-\frac{3 t}{2}} \beta_{n} a_{n, n+1}, \\
e^{-\frac{3 t}{2}} a_{n, n-1} \beta_{n-1}=e^{\frac{3 t}{2}} \beta_{n} a_{n, n-1}, \quad e^{-\frac{3 t}{2}} b_{n, n+3} \beta_{n+3}=e^{\frac{3 t}{2}} \alpha_{n} b_{n, n+3}, \\
e^{-\frac{3 t}{2}} b_{n, n+3} a_{n+3, n+4}=e^{\frac{3 t}{2}} a_{n, n+1} b_{n+1, n+4}, \\
e^{\frac{3 t}{2}} b_{n, n-3} a_{n-3, n-4}=e^{-\frac{3 t}{2}} a_{n, n-1} b_{n-1, n-4}, \\
\alpha_{n} \beta_{n}=\lambda_{n} I_{n} .
\end{gathered}
$$

Последнее уравнение из системы (6.3) позволяет переписать уравнения (6.1)-(6.3) в более компактном виде, исключая матрицу $\beta_{n}$. Мы полагаем также, что матрищы $s^{1}$ и $s^{2}$ симметричны:

$$
a_{i+1, i}=a_{i, i+1}^{T}, \quad b_{i+3, i}=b_{i, i+3}^{T} .
$$

Система уравнений, которую требуется решить для алгебры $G_{2}$, принимает вид

$$
\begin{gathered}
e^{t} a_{n, n-1} \alpha_{n-1}=e^{-t} \alpha_{n} a_{n, n-1}, \quad e^{-t} a_{n, n+1} \alpha_{n+1}=e^{t} \alpha_{n} a_{n, n+1}, \\
e^{\frac{3 t}{2}} b_{n, n-3} \alpha_{n-3}=e^{-\frac{3 t}{2}} \alpha_{n} b_{n, n-3}, \quad e^{-\frac{3 t}{2}} b_{n, n+3} \alpha_{n+3}=e^{\frac{3 t}{2}} \alpha_{n} b_{n, n+3}, \\
-2 e^{t} a_{n, n-1} a_{n-1, n}+2 e^{-t} a_{n, n+1} a_{n+1, n}=\operatorname{sh} t\left(I_{n}-\alpha_{n}^{2}\right), \\
2 e^{3 t} b_{n, n-3} b_{n-3, n}-2 e^{-3 t} b_{n, n+3} b_{n+3, n}=\operatorname{sh} t\left(I_{n}-\beta_{n}^{2}\right), \\
e^{\frac{3 t}{2}} b_{n, n-3} a_{n-3, n-4}=e^{-\frac{3 t}{2}} a_{n, n-1} b_{n-1, n-4} .
\end{gathered}
$$

Здесь, как и в предыдущих разделах, диагональные матрицы $\alpha_{n}$ и $\beta_{n}$ считаются известными из формулы Вейля (1.1); неизвестны прямоугольные матрицы $a$ и $b$ соответствующих размеров.

Как и в случае алгебры $A_{2}$, прямоугольные матрицы $a$ и $b$ имеют блочную структуpy, в терминах примитивных компонент которой можно представить систему (6.4). Мы опускаем это общее рассмотрение, ограничиваясь простейшим примером первого фундаментального представления квантовой алгебры $G_{2}$ с целью показать согласованность предложенной конструкции.

Стандартный базис $(1,0)$-представления с использованием понижающих операторов задается как

$$
\begin{gathered}
X_{1}^{-}|1\rangle, \quad|1\rangle, \quad X_{1}^{-} X_{1}^{-} X_{2}^{-} X_{1}^{-}|1\rangle, \\
X_{2}^{-} X_{1}^{-}|1\rangle, \quad X_{1}^{-} X_{2}^{-} X_{1}^{-} X_{1}^{-} X_{2}^{-} X_{1}^{-}|1\rangle,
\end{gathered}
$$


На таких базисных векторах генераторы $Q=\exp \left[\left(h_{1}+3 h_{2}\right) t\right]$ и $R_{1}, R_{2}$ принимают значения

$$
\begin{aligned}
Q & =\left(e^{2 t}, e^{t}, e^{t}, 1, e^{-t}, e^{-t}, e^{-2 t}\right), \\
R_{1} & \equiv \exp \left(h_{1} t\right)=\left(e^{-t}, e^{t}, e^{-2 t}, 1, e^{2 t}, e^{-t}, e^{t}\right), \\
R_{2} & \equiv \exp \left(3 h_{2} t\right)=\left(e^{3 t}, 1, e^{3 t}, 1, e^{-3 t}, 1, e^{-3 t}\right) .
\end{aligned}
$$

Явные выражения для матриц $\alpha$ имеют вид

$$
\alpha_{1}=e^{-t}, \quad \alpha_{2}=\left(\begin{array}{cc}
e^{t} & 0 \\
0 & e^{-2 t}
\end{array}\right), \quad \alpha_{3}=1, \quad \alpha_{4}=\left(\begin{array}{cc}
e^{2 t} & 0 \\
0 & e^{-t}
\end{array}\right), \quad \alpha_{5}=e^{t} .
$$

Согласно общей схеме ненулевые недиагональные элементы матриц $\left(s^{1}, r^{1}\right)$ определяются формулами

$$
a_{1,2}=(A, B), \quad a_{3,2}=(C, D), \quad a_{3,4}=(E, F), \quad a_{5,4}=(G, H),
$$

а элементы матриц $\left(s^{2}, r^{2}\right)$ - формулами

$$
b_{1,4}=(x, y), \quad b_{5,2}=(u, v),
$$

где все параметры неизвестны и должны быть определены из системы (6.4). После подстановки выражений (6.5) в уравнения из первой строки системы $(6.4)$, получаем

$$
\begin{array}{rlrl}
a_{1,2}=(A, 0), & a_{3,2}=(0, D), & a_{3,4}=(E, 0), & a_{5,4}=(0, H), \\
b_{1,4}=(x, 0), & b_{5,2}=(0, v) .
\end{array}
$$

Из последних двух строк системы (6.4) получаем единственное решение

$$
\begin{gathered}
A^{2}=\operatorname{sh}^{2} t, \quad D^{2}=e^{-t} \operatorname{sh} t \operatorname{sh}(2 t), \\
E^{2}=e^{t} \operatorname{sh} t \operatorname{sh}(2 t), \quad H^{2}=\operatorname{sh}^{2} t, \quad x^{2}=v^{2}=\operatorname{sh}^{2}(3 t) .
\end{gathered}
$$

Прямая проверка показывает, что уравнение в последней строке системы (6.4) вьполненяется автоматически.

\section{7. ЗАКЛЮЧИТЕЛЬНЫЕ ЗАМЕЧАНИЯ}

Результаты данной работы столь же неожиданны, сколь и удивительны, по крайней мере для автора. Долгое время считалось, что решение задачи о явном виде инфинитезимальных генераторов в произвольном представлении должно указывать на глубокую связь с базисами, определенными собственными значениями необходимого числа взаимно коммутируюших операторов, построенных из генераторов соответствуюшей алгебры. Значения операторов Казимира в такой конструкции определяют индексы неприводимого представления. Решение задачи подобным образом было найдено в знаменитых работах Гельфанда и Цейтлина пятьдесят лет назад [2]. Этим авторам удалось явно 
представить инфинитезимальные операторы в произвольном представлении классических полупростых серий $A_{n}, B_{n}$ и $D_{n}$. Однако все многочисленные попытки обобшить эти результаты на оставшиеся полупростые серии были неудачными ${ }^{2)}$. Способ, предложенный в данной статье, не имеет дела ни с операторами Казимира, ни с семейством взаимно коммутирующих операторов и их собственных значений. Существенно только знание результата действия группового оператора $e^{\tau}$ на базисные векторы, и оно дает возможность решить задачу также и для квантовых алгебр.

Полупростые алгебры Ли возникают в пределе, когда параметр деформации стремится к нулю. Обычно мыслится противоположный способ действий: сначала строится представление полупростой алгебры Ли, а затем оно обобшается на случай квантовой алгебры.

Алгоритм данной работы подобен компьютерной программе в том, что необходимо итерировать одну и ту же операцию: решать алгебраическую систему уравнений, неоднородная часть которых известна из предыдущих этапов вычислений. Конечно, ключевым для всей конструкции обстоятельством является формула Вейля для характеров конечномерных неприводимых представлений полупростых групп. Она гарантирует самосогласованность всего построения и дает необходимое число начальных параметров (явные значения диагональных элементов матриц $\alpha, \beta$ и их кратности), через которые выражены генераторы простых корней. Инвариантный характер формулы Вейля позволяет надеяться, что задача о явной реализации генераторов простых корней для квантовых алгебр также может быть решена в инвариантных терминах. К сожалению, эти термины на данный момент автору неизвестны.

Благодарности. Автор благодарит Ч. Девчанда, А. В. Разумова и Н. Вахания за полезные обсуждения и сушественную помошь в подготовке рукописи к печати, а также рецензента за его замечания. Эта работа была выполнена при частичной финансовой поддержке Российского фонда фундаментальных исследований (грант № 98-01-00330).

\section{Список литературы}

[1] Г. Вейль. Классические группы, их инварианты и представления. М.: Гос. изд. иностр. лит-ры, 1947.

[2] И. М. Гельфанд, М. Л. Цетлин. ДАН СССР. 1950. Т. 71. С. 825-829; С. 1017-1020.

\footnotetext{
2) Автор благодарит А. Молева, обратившего внимание на свою статью: А. Молев. MRR № 012-98 (Канберра), где эта проблема была решена для $C_{n}$.
} 